\title{
Theory of analytical curves in atomic fluorescence flame spectrometry
}

\author{
H. P. Hooymayers \\ Physics Laboratory, State University Utrecht, The Netherlands
}

(Received 16 February 1968)

\begin{abstract}
An explicit expression for the intensity of atomic resonance fluorescence as a function of atomic concentration in a flame is derived under certain idealized conditions. The expression is generally valid for a pure Doppler absorption line profile as well as for a combined Doppler and collisional broadened profile (Voigt profile) of the absorption line in the entire range of concentration values.

The expression derived was used to calculate analytical curves for a number of different values of the ratio of collisional line width to Doppler width. The calculations were carried out for a single resonance line in the extreme cases of a very narrow exciting line source and a continuum radiation source, respectively.

Furthermore, a method is given to determine the so called damping parameter " $a$ " and the fluorescence yield factor $p$ from the asymptotic behaviour of the analytical curves if the actual atomic flame content at the intersection point of the initial and final asymptote is calibrated.
\end{abstract}

\section{INTRODUCTION}

Is flame fluorescence spectrometry, the intensity of fluorescent emission is measured when the ground state metal atoms in the flame are excited by the absorption of radiation of the proper frequency emitted by an external background source. The intensity of this re-emission may be used as a measure of the atomic content in the flame.

Winerondnen et al. $[1,2]$ have shown that atomic fluoreseence flame spectrom etry can be successfully used for the determination of several metals in the partper-billion range. Recent investigations by VeiLlon et al. [3] have demonstrated, that not only line sources, but also continuum sources are useful in this method of flame spectrometric analysis.

In order to determine the influence of different absorption parameters on the shape and the relative positions of the analytical curves (i.e. curves in which the fluorescence intensity is plotted vs. metal salt concentration), a detailed theoretical treatment is necessary. Winerondsen et al. [4] have made an initial attempt to derive a general expression for the shape of these analytical curves. However, their formula's do not express the intensity of atomic fluorescence in relevant absorption parameters for the case of a mixed absorption line profile, where collisional broadening is not negligible with respect to Doppler broadening, and no calculated analytical curves are shown in their paper.

[1] J. D. Winefordner and T. J. Vickers, Anal. Chem. 36, 161 (1964).

[2] J. D. Winefordner and T. J. Vickers, Anal. Chem. 36, 1939 (1964).

[3] C. Veillon, J. M. Mansfield, H. L. Parsons and J. D. Winefordxer, Anal. Chem. 38, 204 (1966).

[4] J. D. Winefordner, H. L. Parsons, J. M. Mansfield and W. J. McCarthy, Spectrochim Acta 23B, 37 (1967). 
In this paper, however, a general explicit expression has been derived under idealized conditions for the integrated resonance fluorescence intensity, that is emitted through a small solid angle $\mathrm{d} \omega$ in the direction perpendicular to both the existing light beam and the flame axis (see Fig. 1), as a function of atomic concentration in the flame. From this expression, which is valid for a pure Doppler line profile as well as for mixed line profiles, analytical curves were calculated (see Fig. 2) for a number of different values of the so-called* "a-parameter". For simplicity these calculations are carried out only for a single absorption line (hyper-fine structure is not taken into account) in the limiting cases of a very narrow background line source and a continuum source, respectively.

The exciting line is supposed to traverse the flame as a parallel homogeneous beam with rectangular cross section $\sigma=2 l \times 2 l^{\prime}$, which intersects the flame axis at right angles (see Fig. 1). The dimension $2 l$ of this cross-section is indicated in Fig. 1, whereas $2 l^{\prime}$ represents the dimension of $\sigma$ measured along the direction perpendicular to the plane of drawing. Furthermore, it is assumed that there is a uniform distribution of ground-state atoms throughout the square cross-section of the coloured inner flame. This coloured flame part is supposed to be surrounded by a metal-free burning mantle flame in order to guarantee a uniform temperature distribution in the inner flame. For brevity we will indicate in the following the coloured inner flame by "the flame". Moreover, re-emission of self-absorbed fluorescence radiation will be disregarded.

\section{General Expression for Resonance Fluorescence Intensity}

Under the conditions mentioned in the Introduction, the integrated energy absorbed per second by the layer of absorbing atoms in the element of volume $2 l^{\prime} \mathrm{d} u \mathrm{~d} x$ (see black spot in Fig. 1) can be written as

$$
2 l^{\prime} \mathrm{d} u \mathrm{~d} x \int_{0}^{\infty} I_{\nu} \kappa_{\nu} \exp \left(-\kappa_{v} x\right) \mathrm{d} \nu,
$$

where $I_{v}$ is the radiation flux of the exciting light beam expressed as energy per unit time at frequency $v$ per unit area and per unit frequency interval; $\kappa_{v} \mathrm{~d} x=\beta_{v} N_{0} \mathrm{~d} x$ is the fraction of energy absorbed at frequency $v$ when the exciting light beam travels over a distance $\mathrm{d} x$ in the flame; $N_{0}$ is the ground-state atom concentration in $\mathrm{cm}^{-3}$ and $\kappa_{v}$ is the absorption coefficient (in $\mathrm{cm}^{-1}$ ) at frequency $v$. The intensity of the observed fluorescence radiation $\mathrm{d} E_{F}$ emittcd by this layer in a direction perpendicular to the direction of the exciting light beam through a small (see discussion) solid angle $\mathrm{d} \omega$ is equal to

$$
\mathrm{d} E_{F}=\frac{\mathrm{d} \omega}{4 \pi}\left[2 l^{\prime} \mathrm{d} u \mathrm{~d} x \int_{0}^{\infty} I_{\nu} \mathrm{d} \nu \kappa_{\nu} \mathrm{e}^{-\kappa \nu x}\right]\left[\int_{0}^{\infty} p \alpha_{v^{\prime}} \exp \left\{-\kappa_{v^{\prime}}(L-l+u)\right\}\right] \mathrm{d} v^{\prime},
$$

where $\exp \left\{-\kappa_{v^{\prime}}(L-l+u)\right\}$ accounts for the loss in fluorescence intensity due to

* The damping parameter " $a$ " is proportional to the ratio of collisional line width to Doppler width [5].

[5] C. G. Mrtchell and M. W. Zemansky, Resonance Rad. and Ex. Atoms, 2nd Edition. Cambridge University Press (1961). 
self absorption in the flame; $p$ is the fluorescence yield factor (see Refs. [6-8]) i.e. the fractional probability that an excited atom loses its energy by radiation of a photon before it suffers a quenching collision; $\alpha_{v^{\prime}} \mathrm{d} v^{\prime}$ is the probability of a reemitted photon having a frequency between $v^{\prime}$ and $v^{\prime}+\mathrm{d} v^{\prime}\left(\alpha_{v^{\prime}}\right.$ represents the

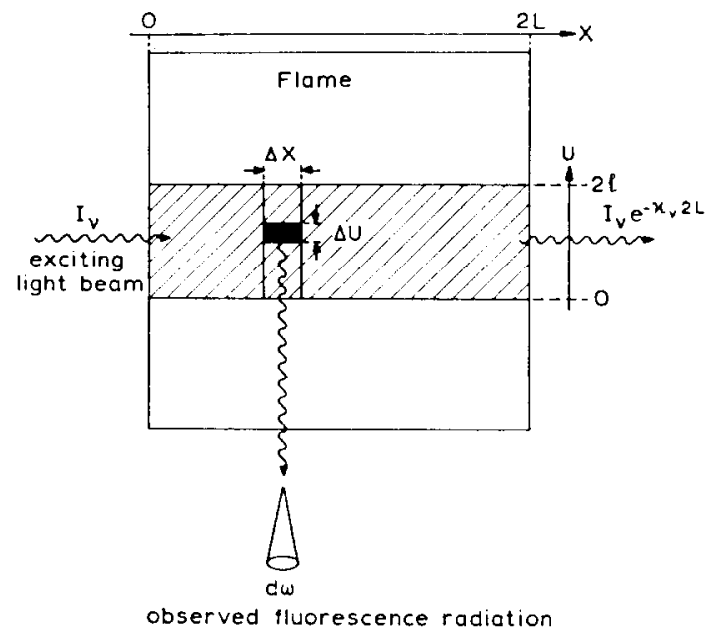

Fig. 1. Part of square flame cross section, as seen from above. The co-ordinate $x$, varying between 0 and $2 L$, measures the distance (in $\mathrm{cm}$ ) from the flame border $(x=0)$ to the considered element of volume $2 l^{\prime} d u d x$ (indicated with a black spot in the figure) along the direction of the exciting beam. $2 l^{\prime}$ is the beam dimension perpendicular to the plane of drawing. The variable $u$, varying between the integration limits 0 and $2 l$, fixes the position of the element of volume in the direction of the observed fluorescence radiation.

normalized shape of the spectral line when not distorted by self absorption, i.e. $\int_{0}^{\infty} \alpha_{v^{\prime}} \mathrm{d} v^{\prime}=1$ ) and $L$ is half the flame thickness. According to the classical theory, we have (5)

$$
\alpha_{v^{\prime}}=8 \pi \frac{1}{\lambda_{0}{ }^{2}} \frac{g_{0}}{g_{1}} \frac{1}{A N_{0}} \kappa_{v^{\prime}}
$$

where $g_{0}$ and $g_{1}$ are the statistical weights of the ground (0) and upper (1) energy levels involved in the absorption transition, respectively; $A$ is the transition probability in $s^{-1}$ for the transition under consideration, $\lambda_{0}$ is the central wavelength of the emitted fluorescence radiation. The validity of this relation can safely be assumed in atmospheric flames since Orthmann and Pringsheim [9] have shown that even under conditions of lower total gas pressure, the frequency distribution of resonance radiation emitted at right angles to the direction of the exciting beam is independent of the spectral shape of the exciting line.

[6] H. P. Hooymayers and C. Тн. J. Alkemade, J. Quant. Spectry Radiative Transfer 6, 510 (1966).

[7] H. P. Hooymayers and C. Th. J. Alkmmade, J. Quant. Spectry Radiative Transfer 6, 847 (1966).

[8] H. P. Hooymayers, Thesis, Utrecht (1966).

[9] W. Orthmann and P. Pringsheim, Z. Phys. 35, 626 (1926). 
Making use of equation (3) and performing the integration over $x$ and $u$ between the limits 0 and $2 L$, and 0 and $2 l$, respectively, we obtain for the total outgoing radiant flux of the fluorescent radiation observed*

$$
\begin{aligned}
E_{F}=\frac{\mathrm{d} \omega}{4 \pi} 2 l^{\prime} p \frac{8 \pi}{N_{0} A \lambda_{0}^{2}} \frac{g_{0}}{g_{1}} \int_{0}^{\infty} I^{\mathrm{v}}\left(1-\mathrm{e}^{-\kappa_{y^{2}} L}\right) \mathrm{d} v \int_{0}^{\infty} \exp \left[-\kappa_{v^{\prime}}(L-l)\right] \\
-\exp \left[-\kappa_{v^{\prime}}(L+l)\right] \mathrm{d} v^{\prime} .
\end{aligned}
$$

We now introduce the dimensionless variable $\xi \equiv \kappa_{0} L\left(=\right.$ const $\left.N_{0} L\right) ; \kappa_{0}$ is the maximum value of the absorption coefficient, $\kappa_{v}$, when Doppler broadening at flame temperature alone is present. We then arrive at the following relation

$$
\begin{aligned}
& E_{F}=\frac{\mathrm{d} \omega}{4 \pi} \sigma p \Delta v_{D}(\pi \ln 2)^{-1 / 2} \frac{L}{l} \frac{1}{\xi} \int_{0}^{\infty} I(y)\{1-\exp (-P(y) 2 \xi)\} \mathrm{d} y \\
& \times \int_{0}^{\infty}\left[1-\exp \left\{-P\left(y^{\prime}\right) \xi\left(1+\frac{l}{L}\right)\right\}-\left(1-\exp \left\{-P\left(y^{\prime}\right) \xi\left(1-\frac{l}{L}\right)\right\}\right)\right] \mathrm{d} y^{\prime}
\end{aligned}
$$

where $y$ stands for the dimensionless frequency difference $\frac{2\left(\nu-v_{0}\right)}{\Delta v_{D}}(\ln 2)^{1 / 2}$; It should be realised, that changing the integration variable $v$ into $y$, the integration limits will change from $(0, \infty)$ into $(-\infty, \infty)$. In this paper however, we have maintained the integration limits $(0, \infty)$ for the variable $y$ by adding a factor 4 to the right hand side of equation (5). In equation (5), $v_{0}$ is the central frequency of the spectral line considered. $\Delta v_{D}=\frac{1}{\lambda_{0}}\left(\frac{8 \pi k T}{m} \cdot \ln 2\right)^{1 / 2}$ is the Doppler half-intensity breadth at flame temperature $T$, and $m$ is the mass of the absorbing atoms in the flame. $\sigma=2 l \times 2 l^{\prime}$ is the cross section of the exciting beam. The value of $\kappa_{0}$ is given by

$$
\kappa_{0}=\frac{2 \sqrt{ }(\ln 2)}{\Delta v_{D} \sqrt{ } \pi} \frac{\lambda_{0}^{2}}{8 \pi} A N_{0} \frac{g_{1}}{g_{0}}, \quad \text { and } \quad P(y)=\frac{\kappa_{v}}{\kappa_{0}}=\frac{a}{\pi} \int_{0}^{\infty} \frac{e^{-t^{2}} \mathrm{~d} t}{a^{2}+(y-t)^{2}},
$$

where $\dagger$

$$
a=\frac{\Delta v_{L}}{\Delta v_{D}}(\ln 2)^{1 / 2}
$$

* This general intensity expression for resonance fluorescence differs from the expression reported by WINEFORDNER et al. [4]. The expression for their factor $f_{z}$ essentially differs from our latter integral in equation (4) for a Voigt profile of the absorption line, though this integral just as $f_{z}$ accounts for the loss in intensity of the fluorescence radiation, when this radiation travels in the flame on its way out. Moreover, it should be noted, that the factor $f_{z}$ introduced by Winefordner et al. is not given in a closed form but as a series expansion, so that even in the case of a pure Doppler line profile, the behaviour of this factor for high metal densities can hardly be investigated, because of the increasingly large number of terms that has to be considered.

$\dagger$ In the definition of the $a$ parameter we have neglected the term $(1 / p)(A / 2 \pi)$, which makes allowance for the broadening effect due to quenching collisions, in comparison to $\Delta v_{L}$ (see Refs. $[5,7])$. 
and $\Delta v_{L}$ is the Lorentz half-intensity breadth (see Refs. [5] and [10]). $P(y)$ describes the normalized spectral shape of an isolated resonance line in an infinitesimal slab of vapour, due to both Lorentz broadening and Doppler broadening. In the case where the absorption line breadth is of the same order as the breadth of the exciting line, the evaluation of $E_{F}$ is difficult and requires detailed information about the frequency distribution of the exciting line, too. However, for the limiting cases of a very narrow line source and for continuum sources such as xenon ares in combination with a monochromator (see VeILLON et al. [3]), the exact profile of the exciting line is not important for the evaluation of $E_{F}$ as a function of $\xi$ and the damping parameter $a$.

\subsection{Case of Continuum Source (source line half-width is large} compared to absorption line half-width)

For a continuum background source, $I(y)$ in equation (5) is virtually constant for all relevant frequences and may be replaced by $I_{0}$, where $I(y)=I_{0}$ for the central frequency of the absorption line in the flame. Then equation (5) may be written as

$$
\begin{aligned}
& E_{F} /\left(\frac{\mathrm{d} \omega}{4 \pi} \cdot I_{0} \sigma \Delta v_{D} /\left(\frac{\pi}{\ln 2}\right) p\right)=\frac{1}{\pi} \frac{L}{l} \frac{1}{\xi} \int_{0}^{\infty}\{1-\exp [-2 \xi P(y)]\} \mathrm{d} y \\
& \quad \times \int_{0}^{\infty}\left[1-\exp \left\{-P\left(y^{\prime}\right) \xi\left(1-\frac{l}{L}\right)\right\}-\left(1-\exp \left\{-P\left(y^{\prime}\right) \xi\left(1-\frac{l}{L}\right)\right\}\right)\right] \mathrm{d} y^{\prime} .
\end{aligned}
$$

The double-logarithmic plot of

$$
E_{F}^{\prime} / \frac{\mathrm{d} \omega}{4 \pi} \cdot I_{0} \sigma \Delta v_{D} /\left(\frac{\pi}{\ln 2}\right) p
$$

as a function of $\xi=$ const. $N_{0} L$ represents the theoretical analytical curve. The analytical curve, defined as the double-logarithmic plot of the meter deflection corresponding to the re-emitted fluorescence radiation versus metal concentration in the sprayed solution, can be obtained from the corresponding theoretical curve by a shift of this curve parallel to the axes of plotting, excluding ionization and other causes of bending.

In the numerical evaluation of the right hand side of equation (6) we used the tabulated results of curve of growth calculations, which were carried out by vas TRIGT et al. [10] with the aid of an electronic binary computer. They have tabulated accurate values (relative error less than $0 \cdot 1$ per cent) of the integral

$$
\int_{0}^{\infty}\left\{1-\exp \left[-\kappa_{0} L P(y)\right]\right\} \mathrm{d} y
$$

in the range $N_{0} f L(\ln 2)^{1 / 2} / \pi \Delta v_{D}=2, \ldots, 9600 \mathrm{~s} \mathrm{~cm}^{-2}$ for a series of $a$-parameter values ranging from $0 \cdot 0$ to $5 \cdot 0$. Here $f$ (which is related to $A$ (Ref. [5]) represents the oscillator strength of the spectral line considered. Using the equality

$$
N_{0} f L(\ln 2)^{1 / 2} / \pi \Delta v_{D}=10 \cdot 63 \xi
$$

[10] C. van Trigt, TJ. Hollander and C. Th. J. Alkemade, J. Quant. Spectry Radiative Transfer 5, 813 (1965). 
which relates the abscissa values used by van Trigt to the quantity $\xi=$ const. $N_{0}$ as introduced in this paper, the theoretical analytical curves are easily computed from van Trigt's results with a relative error of about 0.5 per cent for $0 \leqslant l / L<1$, for all $a$-parameter values used in their calculations, and for $\xi$ ranging from about $0 \cdot 1$ to $10^{3}$. As an example, in Fig. 2 the theoretical analytical curves are plotted for

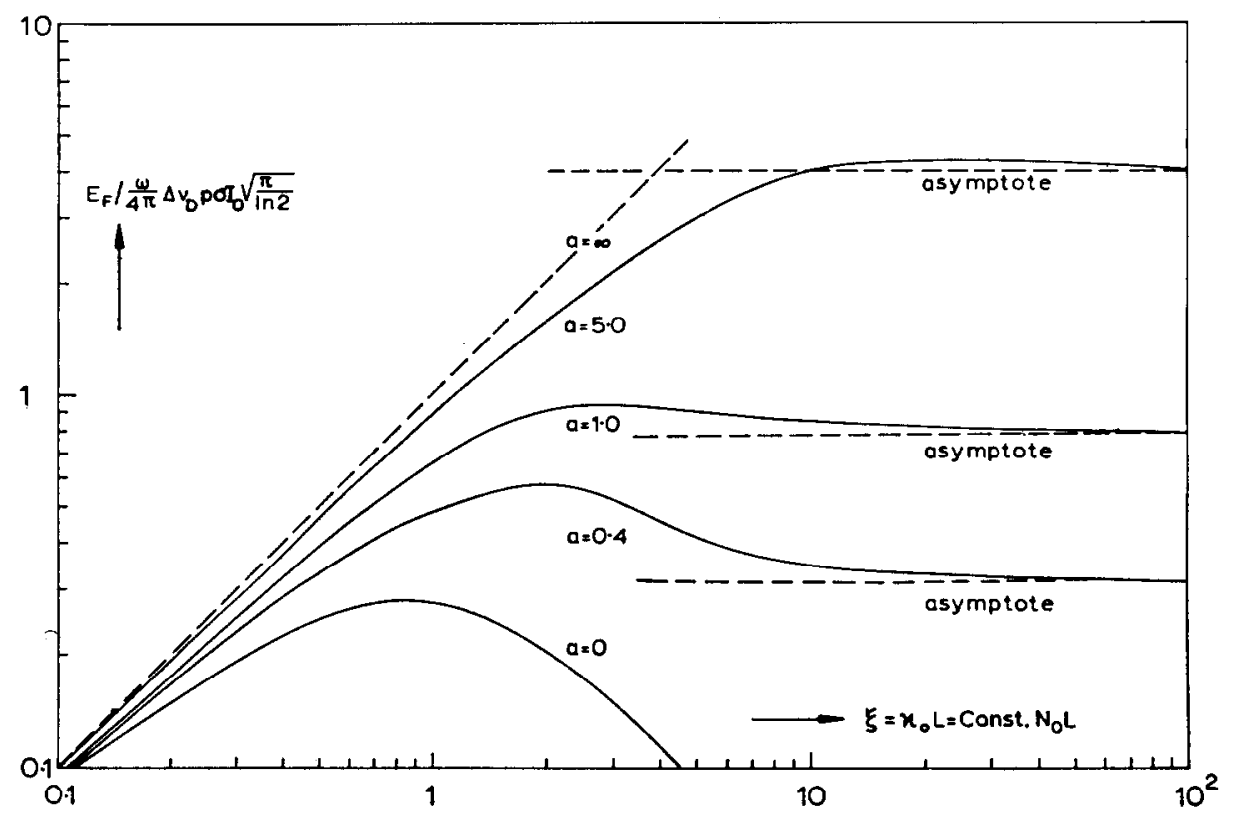

Fig. 2. Some theoretical analytical curves for a single spectral line in the case of a continuum background source. The value of $l / L$ is equal to 0.25 and the $a$-parameter values are $0,0.4,1.0$ and 5.0 respectively (as indicated in the figure). The final asymptotes are calculated from equation (11). The ordinate values in this figure are proportional to the meter deflections corresponding to the fluorescence radiation power while the abscissa values are proportional to the metal concentration $N_{0}$ in the flame.

four different $a$-values and for $l / L=0 \cdot 25$. The corresponding doublet curves are easily computed from these singlet curves, when no overlap occurs between the doublet components [10].

From the plots in Fig. 2 wo learn that $E_{F}$ becomes independent of metal concentration in the high density case, i.e. the analytical curves should reach a horizontal asymptote for an exciting light source with continuum spectrum. This result agrees with the shape of the experimental analytical curves of zinc and cadmium for high atomic densities, as obtained by Vemrov et al. [3] with a $150 \mathrm{~W}$ Xenon arc continuum exciting source.

\section{Low density case}

In the low density limit (small $\xi$ values) $\int_{0}^{\infty}\{1-\exp [-2 \xi P(y)]\} \mathrm{d} y$ can be approximated by $2 \xi \int_{0}^{\infty} P(y) \mathrm{d} y$, while the same type of approximation can be used 
for the self-adsorption term in equation (6). Furthermore we generally have

$$
\int_{0}^{\infty} \kappa_{\nu} \mathrm{d} \nu=\frac{\lambda_{0}^{2}}{8 \pi} \frac{g_{1}}{g_{0}} A N_{0}=\frac{1}{2} \sqrt{\left(\frac{\pi}{\ln 2}\right) \kappa_{0} \Delta v_{D},}
$$

whatever physical processes are responsible for the frequency distribution of the absorption line, so that $\int_{0}^{\infty} P(y) \mathrm{d} y=\sqrt{ } \pi / 2$. In the low density limit equation (6) may thus be replaced by

$$
E_{F} / \frac{\mathrm{d} \omega}{4 \pi} I_{0} \sigma \Delta v_{D} /\left(\frac{\pi}{\ln 2}\right) p \simeq \xi
$$

From equation (8) it can be concluded that in the low density limit, $E_{F}$ should vary linearly with atomic concentration and consequently, under certain conditions (Ref. [10]) linearly with the solution concentration of metal salt introduced into the flame. The latter conclusion agrees with the results of experiments on resonance fluorescence performed by WINEFORDNER et al. [2].

High density case

In the high density limit (large values of $\xi$ ) we may use the well-known approximation

$$
\int_{0}^{\infty}\{1-\exp [2 \xi P(y)]\} \mathrm{d} y \simeq \sqrt{ }(2 \xi a \sqrt{ } \pi)
$$

When inserting this approximation in equation (6) for the absorption term and the same type of approximation for the self-absorption term, equation (6) may be replaced by

$$
E_{F} / \frac{\mathrm{d} \omega}{4 \pi} I_{0} p \sigma \Delta v_{D} \sqrt{ }(\pi / \ln 2) \simeq a /\left(\frac{2}{\pi}\right) \frac{L}{l}\left\{\sqrt{ }\left(1+\frac{l}{L}\right)-\sqrt{ }\left(1-\frac{l}{L}\right)\right\} .
$$

From equation (10) it can be seen, that when $\xi$ tends to infinity, the theoretical analytical curves reach a horizontal asymptote, the ordinate of which is represented by the right hand side of this equation. This outcome agrees with the results of the numerical calculations for $l / L=0 \cdot 25$, presented in Fig. 2 . In the limiting case of $l \mid L=1$ (i.e. the total flame is irradiated by the exciting lamp), the right hand side of equation (10) may be replaced by $2 a / \sqrt{ } \pi$.

Using the series expansion $\sqrt{ }(1+x)=1+\frac{1}{2} x-x^{2} / 2.4+\ldots$ which is valid for $x^{2}<1$, the ordinate value of the final asymptote can be expanded as

$$
\begin{aligned}
a \sqrt{ }\left(\frac{2}{\pi}\right) \frac{L}{l}\left\{\sqrt{ }\left(1+\frac{l}{L}\right)-\sqrt{\left.\left(1-\frac{l}{L}\right)\right\}=a}\left(\frac{2}{\pi}\right)\{1\right. & +\frac{1 \cdot 3}{4 \cdot 6}\left(\frac{l}{L}\right)^{2} \\
& \left.+\frac{1.3 \cdot 5 \cdot 7}{4 \cdot 6 \cdot 8 \cdot 10}\left(\frac{l}{L}\right)^{4}+\ldots\right\} .
\end{aligned}
$$

The leading term of the series expansion approximates the left-hand side of equation (11) with an error tending towards zero when $l / L$ decreases. It should be noted, that 
from this series expansion to $l / L$ one may expect that the theoretical curves for $l / L \leqslant$ 0.5 agree with the curves drawn in Fig. $2(l / L=0.25)$ within a relative error of at most $2 \%$. As a check we found that, when the right hand side of equation 6 was calculated for $l / L=0.1$ and 0.5 respectively, the agreement with the curves plotted in Fig. 2 was indeed within $2 \%$ in the whole range of $\xi$ values considered. Moreover it appears, that for $l / L \leqslant \frac{1}{4}$ the ordinate value of the final asymptote can in good approximation be represented by $a \sqrt{ }(2 / \pi)$ (the relative error is less than $1.5 \%$ ). From this asymptotic value and equation (8) it simply follows that* $\xi_{s}=a \sqrt{ }(2 / \pi)$, where $\xi_{s}$ is the abscissa value of the intersection point of the final and initial asymptote of the theoretical analytical curves [see Fig. 2].

\section{The determination of $a$ and $p$}

The relation $\xi_{s}=a \sqrt{ }(2 / \pi)$ enables us to find the $a$-parameter value, if the actual atomic flame content $N_{0}$ at the intersection point of the asymptotes, $\Delta v_{D}$, and $f$ [see equation (7)] are known or calibrated.

Once the $a$-value has been determined, the value of the fluorescence yield factor $p$ can be found from the ratio of the meter deflection corresponding to the re-emitted radiation power for large atomic densities and the meter deflection (under identical experimental conditions) attained when the phototube is illuminated directly by the exciting light source within monochromator band width of unit frequency interval [see equation (10)]. This procedure for the determination of $a$ and $p$ from the analytical curves is especially suitable when we are interested in $a$ and $p$ values for different resonance lines of the same metal atom in a flame, because then only a single measurement of absolute atomic flame content $N_{0}$ is needed. For the determination of actual atomic flame content $N_{0}$ for a given metal concentration in the sprayed solution it is of advantage to use "the combinatory method" developed by var TRIGT et al. [10]. This method, which is a combination of Hinnov's [11] curve of growth $\uparrow$ method and the duplication curve $\uparrow$ method followed by ALKEMaDE [12], yields both the $a$-value and the actual atomic flame content.

Asymptotic behaviour for $a=0$

For $a=0$, i.e. in the case of a pure Doppler profile of the absorption line, $P(y)$ changes into $\mathrm{e}^{-y^{2}}$. When we use in that case the approximation (Ref. [13])

$$
\int_{0}^{\infty}\left(1-\exp \left[-2 \xi \mathrm{e}^{-y^{2}}\right]\right) \mathrm{d} y \simeq \sqrt{ }(\ln 2 \xi)
$$

which is valid for large $\xi$-values, equation (6) in first approximation may be written

* It should be noted that this relation deviates from the equation (26a) reported in Ref. [4].

$\uparrow$ The "curve of growth" is the (usually double-logarithmic) plot of emission intensity against metal concentration. The "duplication curve" is the (usually double-logarithmic) plot of the "duplication factor" vs. metal concentration; the duplication factor is defined, apart from a normalization factor, as the relative increase in flame emission when a mirror is placed behind the flame.

[11] E. Hinnov and H. КоHN, J. Opt. Soc. Am. 47, 151, 156 (1957).

[12] C. Th. J. AlkkMade, Thesis, Utrecht (1954).

[13] A. UNsöLd, Physik der Sternatmosphären. Springer (1955). 
as

$$
E_{T} / \frac{\mathrm{d} \omega}{4 \pi} \sigma I_{0} \Delta v_{D} /\left(\frac{\pi}{\ln 2}\right) p=\frac{1}{\pi} \frac{L}{l} \frac{1}{\xi} \sqrt{ }(\ln 2 \xi)\left\{/\left[\ln \xi\left(1+\frac{l}{L}\right)\right]-/\left[\ln \xi\left(1-\frac{l}{L}\right)\right] .\right.
$$

In equation (13),

can be replaced by

$$
\sqrt{\left[\ln \xi\left(1 \pm \frac{l}{L}\right)\right]}
$$

$$
\sqrt{ }(\ln \xi)\left(\sqrt{ }\left[1+\frac{\ln \left(1 \pm \frac{l}{L}\right)}{\ln \xi}\right]\right\}
$$

which, in turn, may be approximated for large $\xi$ by

$$
\sqrt{ }(\ln \xi)\left(1+\frac{\ln \left(1 \pm \frac{l}{L}\right)}{2 \ln \xi}\right) .
$$

Inserting this expression in equation (13) and using the first two terms of the series expansion of $\ln (1 \pm l / L)$ we finally find that the right hand side of equation (13) can be reasonably approximated by

$$
(\pi \xi)^{-1} \sqrt{\left[1+\frac{\ln 2}{\ln \xi}\right] \simeq(\pi \xi)^{-1}}
$$

The relative error in this approximation being less than $5 \%$ for $l / L \leqslant 0 \cdot 25$ and $\xi>1000$. In other words for $a=0$ the analytical curve behaves asymptotically as $1 / \xi$ where $\xi$ is, again, proportional to the density of groundstate atoms.

2.2 Case of Narrow Line Source (source line half-width is narrow compared to absorption line half-width)

For an exciting line, the half-width of which is small compared to the absorption line half width, the integral $\int_{0}^{\infty} I_{v}\left\{1-\exp \left[-\kappa_{v} 2 L\right]\right\} \mathrm{d} v$ occurring in the general expression for the intensity of resonance fluorescence [see equation (5)] may be replaced by the approximate expression:

$$
\text { I. . }\left(1-\exp \left[-P_{0} 2 \xi\right]\right) \text {. }
$$

Here $I=\int_{0}^{\infty} I_{v} \mathrm{~d} v$ stands for the integrated (over all frequencies) radiation flux of the homogeneous exciting light beam expressed in energy per unit time and per unit area. Furthermore we have (see Ref. [14])

$$
P_{0} \equiv \frac{\kappa(v=0)}{\kappa_{0}}=\frac{a}{\pi} \int_{-\infty}^{\infty} \frac{\mathrm{e}^{-y^{2}}}{a^{2}+y^{2}} \mathrm{~d} y=\frac{1}{\sqrt{ } \pi} \int_{0}^{\infty} \mathrm{e}^{-a z-z^{2 / 4}} \mathrm{~d} z=\mathrm{e}^{a^{2}}(1-\operatorname{erf} a),
$$

[14] S. S. Penner and R. W. Kavanagh, J. Opt. Soc. Am. 43, 385 (1953). 
where $P_{0}$ is the normalized peak absorption coefficient and erf $a=2 / \sqrt{ } \pi \int_{0}^{a} \mathrm{e}^{-t^{2}} \mathrm{~d} t$ is the well known error function. Substitution of expression (15) in equation (5) yields:

$$
\begin{array}{r}
E_{F} / \frac{\mathrm{d} \omega}{4 \pi} \sigma p 2 I=\frac{1}{2 V^{\prime} \pi} \frac{1}{\xi} \frac{L}{l}\left(1-\exp \left[-P_{0} 2 \xi\right]\right) \int_{0}^{\infty}\left[1-\exp \left\{-P(y) \xi\left(1+\frac{l}{L}\right)\right\}\right. \\
\left.-\left(1-\exp \left\{-P(y) \xi\left(1-\frac{l}{L}\right)\right\}\right)\right] \mathrm{d} y
\end{array}
$$

Using the data tabulated by van TRIGT et al. [10] we also have calculated some theoretical analytical curves in this case of a narrow exciting line source. The calculations were performed for $l / L=0.25$ and for $a=0 ; 1.0$ and 5.0 respectively, and are plotted in Fig. 3.

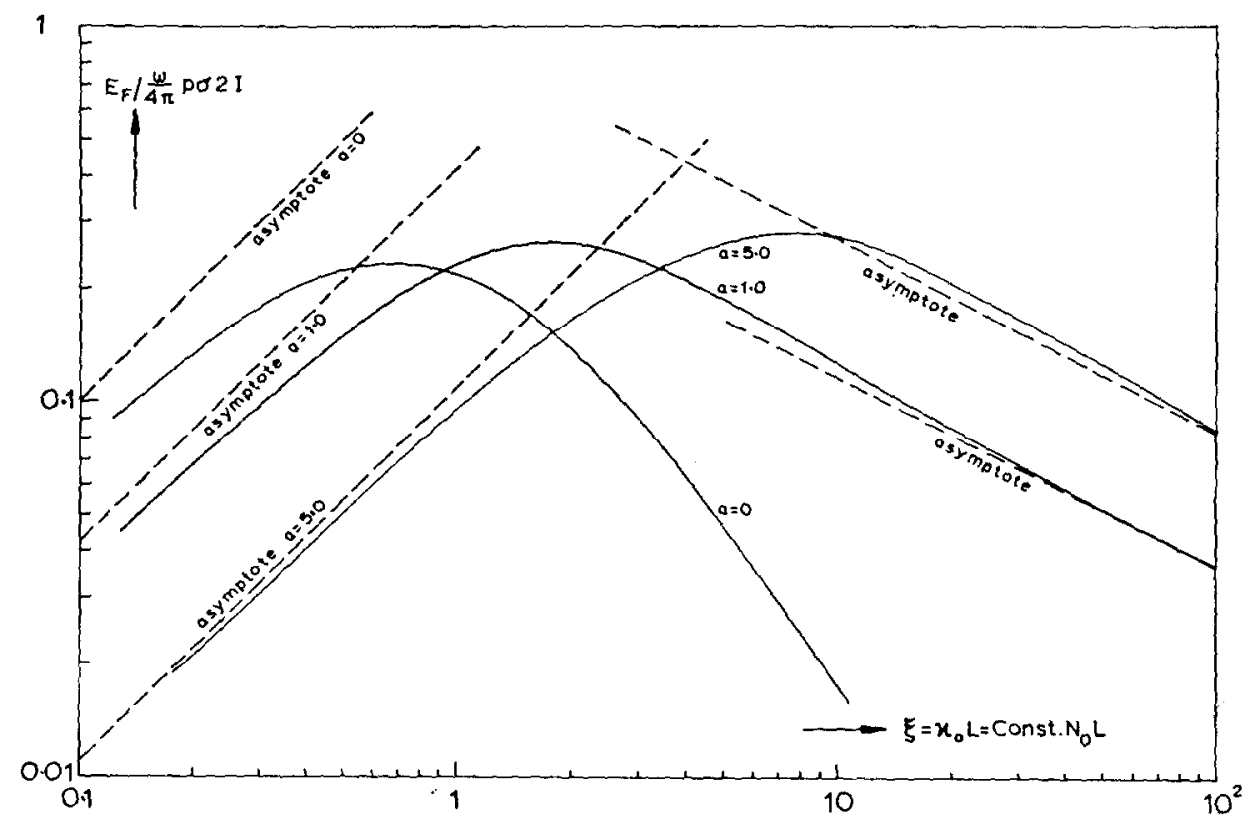

Fig. 3. Some theoretical analytical curves for a single spectral line in the case of a narrow line source. The value of $l / L$ is equal to 0.25 and the $a$-parameter values are $0,1 \cdot 0$ and $5 \cdot 0$ (as indicated in the figure). The initial and final asymptotes are computed from equation (17) and (19), respectively. The ordinate values are proportional to the meter deflections corresponding to the fluorescence radiation power while the abscissa values are proportional to the metal concentration $N_{0}$ in the flame.

Low density case

As low-density asymptote we obtain, considering only the first two terms of the scrios expansion of $\operatorname{cxp}(x)$ :

$$
E_{F} / \frac{\mathrm{d} \omega}{4 \pi} \sigma p 2 I=\xi \cdot P_{0}=\xi e^{a^{2}}(1-\operatorname{erf} a) .
$$


It should be noted, that $\mathrm{e}^{a}(1-\operatorname{erf} a)$ may be approximated by 1 for small $a$-values $(a \leqslant 0 \cdot 1)$ and by $1 / a \sqrt{ } \pi$ for large values of the $a$-parameter $(a>5)$ (see Ref. [15]). Equation (17) shows that in the low density case also for a narrow exciting line the intensity of atomic resonance fluorescence will be proportional to the metal concentration in the flame. Furthermore, it appears that for a small but fixed $\xi$-value, the fluorescence intensity decreases with increasing values of the $a$-parameter. This outcome can be expected, because large $a$-values are associated with broad absorption lines and consequently with small values of the normalized peak absorption coefficient $P_{0}$. The latter conclusion follows from the well-known fact that the integrated absorption $\int_{0}^{\infty} \kappa_{v} \mathrm{~d} v$ is independent of the profile of $\kappa_{v}[5]$.

High density case

In order to obtain the high-density asymptote we use the asymptotic approximation given by equation (9) for the self-absorption portion of equation (16). This leads to the following expression

$$
\begin{aligned}
E_{F} / \frac{\mathrm{d} \omega}{4 \pi} \sigma p 2 I=\frac{1}{2} & \int\left(\frac{a}{\pi^{1 / 2}}\right) \cdot \frac{L}{l}\left[\sqrt{(1+}+\frac{l}{L}\right)-\sqrt{\left.\left(1-\frac{l}{L}\right)\right] \frac{1}{\sqrt{ } \xi}} \\
& =\frac{1}{2} /\left(\frac{a}{\pi^{1 / 2}}\right)\left(1+\frac{(l / L)^{2}}{8}+\ldots\right) \frac{1}{\sqrt{ } \xi} .
\end{aligned}
$$

For $l / L \leqslant \frac{1}{4}$ this equation may be approximated (relative error less than $1 \%$ ) by

$$
E_{F} / \frac{\mathrm{d} \omega}{4 \pi} \sigma p 2 I \simeq \frac{1}{2} /\left(\frac{a}{\pi^{1 / 2}}\right) \frac{1}{\sqrt{ } \xi} .
$$

Realizing that $\xi=$ const. $N_{0}$ and that with narrow line sources, the absorption of exciting radiation approaches $100 \%$ and thus becomes independent of $N_{0}$, we can see from the result that for a constanl amount of absorbed energy, the outgoing resonance fluorescence intensity behaves for $a \neq 0$ as $1 / \sqrt{ } N_{0}$ for $N_{0}$ tending to infinity. For the limiting case of $l / L=1$, equation (18) changes into

$$
E_{F} / \frac{\mathrm{d} \omega}{4 \pi} \sigma p 2 I=\int\left(\frac{a}{2 \pi^{1 / 2}}\right) \frac{1}{\sqrt{ } \xi} .
$$

Combination of the expressions for the initial and final asymptote [see equations (17) and (19)] yields a relation between the abscissa value $\xi_{s}$ of the intersection point of the asymptotes and the $a$-parameter according to $\dagger$

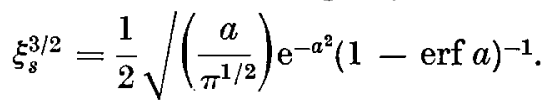

For large $a$-values $\left(a>5\right.$ ) we may use the approximation $\xi_{s}=\left(\frac{1}{2}\right)^{2 / 3} a \pi^{1 / 6}$. For small values of the $a$-parameter the dependence of $\xi_{s}$ on $a$ is rather weak $\left(\xi_{s} \propto a^{1 / 3}\right)$. It can

* The asymptotic behaviour of the theoretical analytical curves for a continum source and $a \neq 0$ can now easily be understood. For in that case, the absorbed primary energy increases for high metal densities with $\sqrt{ } N_{0}$ according to the $\sqrt{ } N$ law, implying that the net intensity of the re-enilled Auorescence radialion becomes independent of $\xi$ (i.e. of metal concentration) as has already been found (see Fig. 2).

$\dagger$ It should be noted that this expression deviates from equation (26b) of Ref. [3].

[15] W. FLÜGGE, Tables of Transcendental Functions. Pergamon Press (1954). 
be read from equation (21) that just as with a continuum source, accurate experimental determination of the mutual position of the asymptotes of the analytical curves will yield the relevant $a$-parameter, if the actual atomic flame content $N_{0}$ is known and vice versa.

\section{Asymptotic behaviour for $a=0$}

In order to understand the asymptotic behaviour of the theoretical analytical curve in the high density case for $a=0$, we once more apply equation (12). This leads for $l / L \leqslant \frac{1}{4}$ and $\xi \geqslant 10^{3}$ to the following asymptotic expression (relative error $\leqslant 5 \%$ )

$$
E_{F} / \frac{\mathrm{d} \omega}{4 \pi} \sigma p 2 I \simeq \frac{1}{2 \sqrt{ } \pi} \frac{1}{\xi \sqrt{ }(\ln \xi)} .
$$

From this equation it can be seen that in the case of high metal densities $E_{F}$ varies as $1 /[\xi \sqrt{ }(\ln \xi)]$ which is nearly similar to the asymptotic behaviour of the analytical curve for $a=0$ in the case of a continuum exciting source [see equation (14)], since $\sqrt{ }(\ln \xi)$ varies very slowly with $\xi$.

\section{Discussion}

It should be noted, that we used in our calculations the assumption that the profile of the emission coefficient $\alpha_{v}$ is identical to the profile of the absorption coefficient $\kappa_{v}$. This is only true when the frequency at which a photon is absorbed and the frequency at which it may be re-emitted are uncorrelated. One may expect that this condition is fulfilled when an excited atom suffers many perturbing adiabatic collisions during the actual radiative lifetime. From our earlier fluorescence experiments we know that the average number of such collisions per actual radiative lifetime lies between 15 and 120 for usual flames under atmospheric pressure [5, 7].

From these results we learn that the assumption concerning the profile of $\alpha_{v}$ and $\kappa_{v}$ can safely be made.

\section{Consideration of the restrictions as to solid angle $\mathrm{d} \omega$}

In our calculations of the analytical curves for a continuum source and a narrow line source, we have made the assumption, that the fluorescence radiation is emitted through a small solid angle $\mathrm{d} \omega$ in the direction of the detector. In order to check whether this assumption is crucial or not, we consider a hypothetical experimental situation where the solid angle under which the detector views the flame is not actually small.

Assume, for instance, that $l / L \rightarrow 0$ and that the angle between the extreme rays entering the detection lens, is about 30 degrees. Then these extreme rays will travel over a distance $1.03 \mathrm{~L}$ in the flame before leaving the flame. In the high-density case this will result in values for $E_{F}$ for these extreme rays, which are about $1.5 \%$ less than those presented in Figs. 2 and 3 for rays parallel to optical axis. The deviation between the actual and plotted $E_{F}$ values tends towards zero when $N_{0}$ decreases.

Acknowledgements-The author is indebted to Professor C. TH. J. Alkemade for discussion of this work and his valuable suggestions and to Professor J. D. WINEFordner for reading this paper carefully and for his useful comments. 